\title{
Cardiovascular safety of central nervous system stimulants in children and adolescents: population based cohort study
}

\author{
(c) $(1)$ (8) OPEN ACCESS
}

\begin{abstract}
Almut G Winterstein professor ${ }^{1}$, Tobias Gerhard assistant professor ${ }^{2}$, Paul Kubilis statistical research coordinator ${ }^{1}$, Arwa Saidi professor ${ }^{3}$, Stephan Linden research assistant ${ }^{1}$, Stephen Crystal board of governors professor ${ }^{4}$, Julie Zito professor ${ }^{5}$, Jonathan J Shuster professor ${ }^{6}$, Mark Olfson professor ${ }^{7}$

${ }^{1}$ Pharmaceutical Outcomes and Policy, College of Pharmacy, Epidemiology, Colleges of Medicine and Public Health and Health Professions, University of Florida, Gainesville, Florida 32611, USA; ${ }^{2}$ Ernest Mario School of Pharmacy and Institute for Health, Health Care Policy, and Aging Research, Rutgers, State University of New Jersey, New Brunswick, NJ 08901, USA; ${ }^{3}$ Pediatric Cardiology, College of Medicine, University of Florida, Gainesville, Florida, ; ${ }^{4}$ Center for Health Services Research on Pharmacotherapy, Chronic Disease Management, and Outcomes, Rutgers University, New Brunswick; ${ }^{5}$ University of Maryland, Baltimore, MD 21201, USA; ${ }^{6}$ Department of Health Outcomes and Policy, UF Clinical Research Center, College of Medicine, University of Florida, Gainesville, Florida; ${ }^{7}$ Department of Psychiatry, Columbia University, New York State Psychiatric Institute, New York, NY 10032, USA
\end{abstract}

\begin{abstract}
Objectives To evaluate the cardiac safety of central nervous system stimulants in children and adolescents.

Design Population based retrospective cohort study.

Setting Automated healthcare claims data from 1219847 children and young people eligible for 28 state Medicaid programmes from 1999 to 2006 linked to the Social Security Death Master File and the National Death Index.

Participants Children and young people age 3-18 entered the cohort at the first diagnosis of a mental health condition commonly treated with stimulants (such as attention-deficit/hyperactivity disorder) after a minimum period of six months' eligibility and were followed until loss of eligibility, their 19th birthday, admission to hospital for longer than 30 days, or death. Exclusion criteria included transplant recipients, receipt of dialysis, or claims indicating substance misuse. We retained high risk groups with similar use of stimulants as low risk children (such as children with congenital heart disease). Sociodemographic characteristics, cardiac risk factors, and psychiatric diagnoses obtained from before the index period were summarised with a propensity score. We used discrete survival analysis to estimate the relative risk for periods of stimulant use and non-use, adjusted for propensity score and antipsychotic use for the full cohort and the high risk and low risk groups.
\end{abstract}

Main outcome measures Composite endpoint of stroke, acute myocardial infarction, or sudden cardiac death; a secondary composite endpoint added ventricular arrhythmia
Results A total of 66 (95 including ventricular arrhythmia) events occurred during 2321311 years of follow-up. The odds ratio adjusted for propensity score and antipsychotic use for current versus no stimulant use was 0.62 (95\% confidence interval 0.27 to 1.44$)$, with a corresponding adjusted incidence rate of 2.2 and 3.5 per 100000 patient years for current stimulant and non-use, respectively. Twenty six events occurred in high risk patients (incidence rate 63 per 100000 patient years) with an odds ratio of 1.02 (0.28 to 3.69$)$. Odds ratios for the secondary endpoint were similar to those for the primary endpoint $(0.74$, 0.38 to 1.46$)$

Conclusions Treatment of children with central nervous stimulants is not significantly associated with an increase in the short term risk of severe cardiac events. Analyses cannot be generalised to children with long term use of stimulants. Furthermore, long term effects of slight increases in heart rate or blood pressure are unknown.

\section{Introduction}

The past decade has seen clinical and policy debates over the potential cardiac risk of central nervous system stimulants approved for the treatment of attention-deficit/hyperactivity disorder (ADHD). The paucity of conclusive evidence regarding safety of stimulants has resulted in recommendations in favour of and against a black box warning, ${ }^{12}$ withdrawal and reintroduction of Adderall (mixed amphetamine salts) by the Canadian regulatory agency, and controversy regarding the need for electrocardiography before the start of treatment. ${ }^{34}$ Concerns 
were based on case reports of sudden cardiac death in stimulant users, plausible pharmacological pathways involving well established stimulant effects on heart rate and blood pressure, ${ }^{5-9}$ and a limited number of observational studies too small to examine serious cardiac events. ${ }^{10-13}$

A recently published study from a population of 1.2 million predominantly privately insured young people compared cardiac risk among stimulant users and a matched sample of non-users. ${ }^{14}$ This study found a low incidence of sudden cardiac death, acute myocardial infarction, and stroke, and no association between these serious cardiovascular events and stimulant use (hazard ratio $0.75,95 \%$ confidence interval 0.31 to 1.85 ). The study was preceded by a similar smaller study of publicly and privately insured children in the United States that reported an inconclusive hazard ratio of $1.60(0.19$ to 13.60$)$ for the primary endpoint. ${ }^{13}$

We report the results of the second US federally funded study of the effects of stimulants on major cardiovascular events. We assembled a population based cohort of publically insured children and young people from 28 State Medicaid programmes (representing about $82 \%$ of the US fee for service Medicaid population in 2002). Non-users of stimulant had to have and maintain a diagnosis of attention-deficit/hyperactivity disorder, or other mental health diagnosis with a high propensity for stimulant treatment, to establish similar propensity for psychiatric care and thus more balanced comparison groups. On the basis of a stipulated sympathomimetic pathogenesis mediated by chronotropic effects, we evaluated the risk for ventricular arrhythmia in addition to major cardiovascular events. Finally, based on the observation that serious diseases such as sickle cell anaemia or congenital heart defects do not preclude a diagnosis of attention-deficit/hyperactivity disorder or treatment in our cohort, we relaxed exclusion criteria and have reported results stratified by risk status to evaluate cardiac safety in the most vulnerable population. Cardiovascular risk associated with stimulants could be of particular concern in these groups of patients.

\section{Methods}

\section{Study population}

The study cohort was assembled from the 28 US states with the largest paediatric population eligible for Medicaid fee for service benefits in 1999-2006, representing 24 million children and young people aged 0-18. The Medicaid Extract Files (MAX), assembled by the Centers for Medicare and Medicaid Services, provide monthly detail on Medicaid eligibility and claims for all inpatient and outpatient services (along with diagnoses and procedures) and drugs reimbursed by Medicaid. We ascertained information on deaths from respective entries in the Medicaid eligibility files as well as through linkage with the Social Security Death Master File. Social security numbers and date of birth of flagged participants were then forwarded to the National Death Index for confirmation and ascertainment of cause of death.

In this retrospective cohort study participants entered the cohort at the first diagnosis of attention deficit hyperactivity disorder (ICD-9-CM (international classification of diseases, ninth revision, clinical modification) code 314.0x), adjustment reaction (code 309.2x), disturbance of conduct (code 312.xx except $312.3 \mathrm{x}$ ), or other mixed or unspecified emotional disturbance (codes 313.8x, 313.9). Participants had to have at least six months' continuous eligibility before the first qualifying diagnosis (index date) and be aged at least 3 and not more than 18 at the index date.
Children or young people were excluded if they had an inpatient or outpatient claim with any diagnoses of the study endpoints (see appendix, table A) or any stimulant use in the six months before the index date. We also excluded children with any claim indicating organ transplant status (ICD-9-CM codes V42.xx, V43.2x, V43.3x, V43.4x, V49.83) or dependency on dialysis (code V56.0) because stimulant use was minimal. Any indication of substance use disorder also served as exclusion criterion because we could not capture alternative causes for cardiac events. We excluded patients with any drug claim for pemoline, methamphetamine, or monoamine-oxidase (MAO) inhibitors at any time during the study period and with index date of 1999-2002 in Tennessee and 1999 to June 2000 in Pennsylvania because of incomplete capture of claims for prescription drugs during this time period.

We flagged several high risk disease states for stratified analysis rather than exclusion because rates of stimulant use were similar to those in low risk categories. Definition of high risk for serious cardiovascular events included a diagnosis of malignant neoplasm, hereditary or acquired haemolytic and aplastic anaemia, HIV or AIDS, congenital heart disease or other congenital disease with vascular involvement, cardiomyopathy, valve disorders, or cerebrovascular disease (see appendix, table B).

Participants were followed until the end of eligibility for Medicaid fee for service benefits, an admission to hospital of more than 30 days, their 19th birthday, death, a claim indicating pregnancy, or lack of subsequent qualifying mental health diagnosis within 24 months of the previous diagnosis. For sensitivity analysis we introduced an additional set of censoring diagnoses involving trauma (such as head injury) or acute inflammatory heart disease or intracranial or severe viral infections, which can acutely increase the risk for cardiovascular events (see appendix, table C).

\section{Study endpoints}

We used two composite study endpoints: sudden cardiac death, acute myocardial infarction, and stroke; and sudden cardiac death, acute myocardial infarction, stroke, and ventricular arrhythmia. The first endpoint follows the definition of the primary endpoint reported by Cooper et al (see appendix, table A) $)^{14}$ and has been validated against medical charts, death certificates, and autopsy reports (positive predictive value of 91\%). Our second endpoint added admissions to hospital with a principal diagnosis of ventricular arrhythmia (ICD-9-CM 427.1 or 427.4 or 427.41 or 427.42 ) to the primary endpoint to capture severe arrhythmia as a result of chronotropic effects.

\section{Stimulant exposure}

Stimulant use was defined on the basis of pharmacy claims for methylphenidate and mixed amphetamine salts with a start at the day of dispensing. The end date for each claim was estimated based on the dispensed supply in days plus a $25 \%$ grace period to incorporate residual supply because of drug holidays (such as during weekends). A new dispensing was set to over-ride previous assignments of exposure without adjustments for potential overlaps between time periods that were covered by stimulants based on the dispensed days' supply. About $85 \%$ of claims were for a 30 day supply, resulting in 38 day exposure periods for any of these claims. We conducted sensitivity analyses with no grace period and a 50\% grace period. Periods after current use were defined as former use. No use included the entire follow-up period of never users and the period preceding the start of drug treatment among stimulant users. 


\section{Covariates}

We used logistic regression to calculate exposure propensity scores to estimate the likelihood of receiving stimulants. ${ }^{16}$ Covariates, measured during the six month period before the index date, included sex, race/ethnicity, year, state of residence and age at index date, reasons for eligibility for Medicaid (such as foster care, receipt of temporary assistance for needy families, disability), other psychotropic drug classes, drug classes typically used for cardiovascular disease or to treat cardiovascular risk factors, cardiac diseases and conditions that predispose to cardiovascular disease, and various mental disorder diagnoses (see appendix, table D for operational definitions). We also included designated high risk conditions such as congenital heart disease for the main analysis but removed them for the risk stratified analysis. Participants were weighted by the inverse of their propensity score to evaluate the level of balance achieved between exposed and unexposed groups across all baseline covariates.

To account for concomitant use of drugs that might cause cardiovascular events we also developed time dependent covariates for use of other sympathomimetic drugs (predominantly prescribed oral decongestants and inhaled $\beta$ agonists), $\alpha$ agonists, antipsychotics, and antidepressants. Calendar month and age (modelled linearly or by restricted cubic spline ${ }^{17}$ ) were also included as time dependent variables in the risk models.

\section{Data analysis}

We used a discrete time survival analysis framework to compare the odds of study endpoints occurring within periods of current versus no stimulant use as well as current versus former use. This proportional odds model is analogous to the Cox proportional hazards model. ${ }^{18}{ }^{19}$ For computational efficiency, we segmented follow-up time into 15 day increments and assigned exposure status based on the majority of days attributed to current, former, or no use of stimulants within each segment. We applied the same majority rule to designate all other time dependent covariates. We used restricted cubic splines ${ }^{17}$ to explore the trend shapes of the baseline hazard function, ${ }^{20}$ the logit transformed propensity score, and time dependent age at the start of each 15 day segment. This analysis indicated flat trends for age and the baseline hazard function (that is, a constant hazard rate) and a linearly decreasing trend for the logit transformed propensity score in all risk models. Covariates were retained in a risk model if they changed the point estimate of stimulant risk by more than $10 \%$, as recommended for model building in epidemiological studies. ${ }^{21} \mathrm{We}$ computed separate regression models for the full cohort and the two risk strata before and after censoring patients because of trauma or acute infections. Study endpoints were rare enough to consider the exposure odds ratio to be a reasonable estimate of relative risk. ${ }^{22}$ Adjusted incidence rates were therefore calculated by multiplying the crude incidence rate of non-use by the respective adjusted odds ratio. We used the independent sample $t$ test to compare mean age between exposed and unexposed groups and Pearson's $\chi^{2}$ test to compare proportions of other covariates. Data management and analysis were conducted with SAS version 9.2 (SAS Institute, Cary, NC). Smoothed density curves for propensity score distributions were estimated and plotted with R version 2.14.1 (R Foundation for Statistical Computing, Vienna, Austria).

\section{Results}

The cohort included 1219847 children and young people with a new diagnosis of attention-deficit/hyperactivity disorder, adjustment reaction, disturbance of conduct, or emotional disturbance disorder (386 584 with at least one claim for stimulants) with a total of 2321311 person years of follow-up (figure $\Downarrow$ ). Follow-up time was distributed as follows: 1506877 person years of non-use, 416018 of current stimulant use, and 398416 years of former stimulant use. The mean and median follow-up time for the whole cohort was 1.9 years and 1.6 years, respectively. For young people who used stimulants, the mean and median time on stimulants (current use) was 1.1 and 0.6 months. The most common reasons for censoring were loss of eligibility (84.6\%), lack of recurrent diagnosis of ADHD within 24 months $(10.1 \%)$ and participant's 19th birthday (3.5\%). A total of $1010(0.08 \%)$ children died from causes other than those included in the study endpoints.

\section{Use of stimulants}

Consistent with previous reports, white children/young people and males were over-represented among stimulant users (table 1) $\downarrow$, while use of antipsychotics or antidepressants and diagnoses of depression, bipolar disorder, anxiety, or schizophrenia were more common among non-users (table $2 \Downarrow$ ). ${ }^{23}{ }^{24}$ Of note, similar proportions of children with high risk conditions such as congenital heart disease were represented among users and non-users. If considered together, non-users had slightly higher rates of cardiovascular risk factors, but differences were subtle.

Accordingly, distributions of propensity scores were similar among users and non-users (see appendix fig A). Inverse weighting of participants by their exposure propensity score established balanced groups with a maximum of $1 \%$ crude difference in the distribution of covariates among stimulant users and non-users (tables 1 and 2).

\section{Cardiovascular risk of stimulants}

There were 66 cases of sudden cardiac death, stroke, or acute myocardial infarction, resulting in an overall event rate of 2.8 per 100000 patient years. Unadjusted event rates in the full cohort were 3.5 per 100000 patient years for non-users of stimulants, 1.7 for periods of current use, and 1.5 for periods of former use. The adjusted odds ratio for current versus no stimulant use was 0.62 (95\% confidence interval 0.27 to 1.44 ), with a corresponding adjusted incidence rate of 2.2 per 100000 patient years for current stimulant use compared with non-users (tables $3 \Downarrow$ and $4 \Downarrow$ ). Besides the propensity score that summarised all fixed covariates, the only time dependent covariate that we included in the final model because of its significant effect on the risk estimate for current stimulant use was antipsychotic use with an odds ratio of 3.44 (1.68 to 7.03). The adjusted odds ratio for the comparison of current use of stimulants versus former use was 1.07 (0.36 to 3.21$)$.

Twenty six events occurred in high risk patients, who contributed only 40991 patient years with a crude incidence rate of 63 per 100000 patient years. Stimulant use was not associated with an increased risk of cardiovascular events in either group, although confidence intervals were wide (0.49 ( 0.16 to 1.48$)$ and 1.02 ( 0.28 to 3.69$)$ for low risk and high risk, respectively).

Addition of admissions to hospital for ventricular arrhythmia increased the total number of events to 95 but showed similar odds ratios for this composite endpoint, both for the full and stratified cohorts. Censoring for trauma or acute infection 
reduced the number of events considerably but similarly had little effect on risk estimates. Finally, analyses within stimulant users only showed no difference for the comparison of periods of current versus former use, but confidence intervals were wide because of the small number of events.

\section{Discussion}

Our study supports previous findings confirming the safety of central nervous system stimulants in the treatment of attention-deficit/hyperactivity disorder and other mental disorders in children and young people. Similar to the report by Cooper and colleagues, most point estimates were below 1, indicating a small chance for excess risk associated with stimulant use and suggesting a residual healthy user bias. ${ }^{14}$ The upper confidence limits for the full cohort suggest that the maximum increase in risk should be no be higher than $44 \%$ for sudden cardiac death, acute myocardial infarction, or stroke and $46 \%$ for these events or ventricular arrhythmia, respectively, a lower upper risk boundary than reported in the study by Cooper and colleagues (hazard ratio $0.75,0.31$ to 1.85 ).${ }^{14}$ With a baseline incidence rate of less than three events per 100000 patient years of stimulant use, the absolute increase in risk, if any, can be expected to be small.

Stratified analysis did not support a differential effect of stimulants in young people at high risk and emphasised, as observed by others, that these events are extremely rare in healthy children. ${ }^{10}{ }^{12-14} 25 \mathrm{~A}$ small fraction of high risk patients, however, accounted for close to half of all events, suggesting a profound difference in risk. Of note, our ability to identify high risk status was dependent on respective diagnoses in automated claims data and some high risk patients might have been missed. Finally, inclusion of admissions to hospital for ventricular arrhythmia did not change our results. We conducted additional analyses with expanded definitions of admission for arrhythmia and cardiomyopathy, resulting in several hundred events, but risk estimates remained essentially the same and incidence rates were consistently low (results not shown). Because the claims based definitions of these endpoints have not been sufficiently validated in children our results should be interpreted with caution.

On the basis of US census estimates our study cohort was drawn from a population that represented more than a quarter of all children in the US. Nevertheless, in common with the previous studies, it struggled with small event rates, despite a massive sample size, and further emphasises periods of use of stimulants in this population. Because of its focus on children eligible for public health insurance (providing coverage for low income families, children in foster care, or children with disabilities and over-representing minorities) our study allowed evaluation of a more vulnerable population when compared with children with private insurance. Rather than excluding high risk groups, we present stratified risk estimates for high risk conditions associated with frequent stimulant use. The overall incidence rate for the primary endpoint (2.8 per 100000 patient years) was similar to that in the study by Cooper and colleagues ( 3.1 events for 100000 patient years). ${ }^{14}$ Our slightly lower event rates might be because of restriction to children with select mental health diagnoses, which was not required by Cooper and colleagues. ${ }^{14}$ That study also used a broader claims based screening algorithm to ascertain events if patient charts and autopsy reports were retrievable for verification, while our study relied on their validated automated case definitions.

\section{Study strength and limitations}

Our study has several distinct strengths. Firstly, we used a new user design because of our concern that immediate sympathomimetic effects such as tachycardia might cause early discontinuation of treatment in children more susceptible to cardiac events. ${ }^{26}$ Secondly, several features aided in establishing a balanced comparison group, including the requirement for diagnoses of mental disorder with high propensity for treatment with stimulants and the broad selection of variables for propensity score adjustment. Our comparison of baseline characteristics suggests that non-users had slightly more cardiovascular risk factors, but the magnitude of confounding was small, which is reflected in well overlapping propensity score distributions among stimulant users and non-users (see appendix, fig A). Use was largely associated with demographic variables and psychiatric parameters, which is consistent with previous reports. ${ }^{27} \mathrm{We}$ might not have been able to capture the full range of cardiac risk factors from claims data and stimulant users might have been generally healthier. Our observation that the presence of even severe cardiac risk factors did not preclude treatment with stimulants, however, increases our confidence that the impact of residual confounding is probably limited - that is, that odds ratios are reversed because of its effects.

Furthermore, our previous analysis in young people eligible for Florida Medicaid, a subpopulation of this study cohort, found a $20 \%$ increase in emergency department visits for mild cardiac causes, congruent with clinical trial reports of increased heart rate and blood pressure..$^{10}$ The fact that these mild effects were captured in that analysis reduces the likelihood that a healthy user effect masked stimulant cardiac effects. Thirdly, even though our study was not designed to examine risks associated with antipsychotic use, it showed a significant cardiac risk for these agents of similar size as reported previously in adults. ${ }^{28} 29$ Finally, we aimed to exclude alternative causes, which could bias results, especially when event rates are as low as we observed. For example, the study by Schelleman and colleagues, which had included admissions to hospital and emergency department visits with a primary diagnosis of cardiac arrest, found low positive predictive values for their composite endpoint. ${ }^{13}$ When we examined causes of death attributed to these events in death records we noted a large proportion with non-cardiovascular causes, especially trauma. Thus, we relied on a validated claims based definition for our primary endpoint. We further confirmed our findings with a sensitivity analysis that censored children when claims indicated severe injury and acute infections.

As with all claims based studies, we cannot exclude non-adherence, which would result in misclassification of users and underestimate the risk of stimulants. Because most states permit only 30 days' supply for controlled substances, however, exposure information was often updated according to prescription refills. We furthermore did not see any impact when we changed the definition of our grace period that defined the lengths of each prescription (data not shown). We also examined whether the use of majority rules in assigning drug exposure to the 15 day increments in our analysis might have introduced misclassification. For this, we examined the attribution of all adverse events to exposure status on the basis of definitions of daily versus 15 day exposure. The attribution for all events matched the exposure status that was assigned based on the 15 day period majority rules. We also ran sensitivity analyses that excluded time periods when participants were admitted to hospital in the short term (less than 30 days) for reasons other than the study endpoints, which our primary analysis ignored. 
Results were virtually identical. Finally, illicit use of stimulants in non-users might have contributed to misclassification.

With a median follow-up time of about two years our study is not able to examine the safety of long term use of stimulants. A possible pathway of severe short term effects is cardiomyopathy induced by tachycardia, a reversible form of cardiomyopathy that has been described in children and adults. ${ }^{30}$ Control of arrhythmias, which usually present as supraventricular or ventricular arrhythmia, and heart rate usually results in rapid improvement in cardiac function with normalisation of the ejection fraction within one to two weeks. ${ }^{32}$ The effect of even slight increases in heart rate or blood pressure over many years, however, is unclear. Likewise, it is unclear whether the effect of such subtle increases could manifest many years after use of stimulants.

Finally, because of sample size constraints we could not provide comparative safety estimates for different doses of stimulants or for methylphenidate and mixed amphetamine salts separately, but, given the small overall incidence of severe cardiac events, differences are expected to be subtle. We also did not find indications for differential effects in our previous analysis of emergency department visits resulting from cardiac symptoms. ${ }^{10}$

\section{Conclusions}

We examined a different, more vulnerable population than previous studies, and have confirmed earlier findings that treatment of children with central nervous stimulants is not associated with an increase in risk of severe cardiac events. Even if general concerns about bias in observational studies are considered, the observed incidence rates provide assurance for minimal absolute risk. It is important to note, however, that stimulant use was short term and the results might not generalise to children who use stimulants over many years. Furthermore, long term effects of slight increases on heart rate or blood pressure, even after discontinuation of use, are unknown and require ongoing monitoring for safety.

Contributors: AGW and TG made substantial contributions to study conception, AGW, SL, and PK were responsible for data acquisition. AGW, TG, and PK were responsible for analysis of data. All authors contributed to study design and interpretation of data. AGW drafted the article and is guarantor. All authors provided critical revisions for important intellectual content and approved the final version.

Funding: This study was funded by grant R01-HS0185606 from the Agency of Healthcare Research and Quality (AHRQ) and in part by NIH grant 1UL1 TR000064 from the National Center for Advancing Translational Sciences. AHRQ and NIH had no role in the design, conduct, or reporting of the study or in the decision to submit the manuscript for publication.

Competing interests: All authors have completed the ICMJE uniform disclosure form at www.icmje.org/coi_disclosure.pdf (available on request from the corresponding author) and declare that $\mathrm{MO}$ has received funding from the National Institute for Mental Health for a related topic.

Ethical approval: The study was approved by the University of Florida institutional review and privacy boards (\#557-2009) and the Centers for Medicare and Medicaid Services privacy board (DUA 20929).

Data sharing: Data were obtained under data users agreements with the Centres for Medicare and Medicaid and the National Death Index and are not available for sharing.

1 Nissen SE. ADHD drugs and cardiovascular risk. N Engl J Med 2006;354:1445-8.
2 Perrin JM, Friedman RA, Knilans TK, Group BBW, Surgery SoCaC. Cardiovascular monitoring and stimulant drugs for attention-deficit/hyperactivity disorder. Pediatrics 2008;122:451-3.

3 Vetter VL, Elia J, Erickson C, Berger S, Blum N, Uzark K, et al. Cardiovascular monitoring of children and adolescents with heart disease receiving medications for attention deficit/hyperactivity disorder [corrected]: a scientific statement from the American Heart Association Council on Cardiovascular Disease in the Young Congenital Cardiac Defects Committee and the Council on Cardiovascular Nursing Circulation 2008;117:2407-23.

4 Bélanger SA, Warren AE, Hamilton RM, Gray C, Gow RM, Sanatani S, et al. Cardiac risk assessment before the use of stimulant medications in children and youth. Paediatr Child Health 2009;14:579-92.

5 Biederman J, Mick E, Surman C, Doyle R, Hammerness P, Harpold T, et al. A randomized, placebo-controlled trial of OROS methylphenidate in adults with attention-deficit/hyperactivity disorder. Biol Psychiatry 2006:59:829-35

6 Weisler RH, Biederman J, Spencer TJ, Wilens TE. Long-term cardiovascular effects of mixed amphetamine salts extended release in adults with ADHD. CNS Spectr 2005;10:35-43.

7 Samuels JA, Franco K, Wan F, Sorof JM. Effect of stimulants on 24-h ambulatory blood pressure in children with ADHD: a double-blind, randomized, cross-over trial. Pediatr Nephrol 2006;21:92-5.

8 Stowe CD, Gardner SF, Gist CC, Schulz EG, Wells TG. 24-hour ambulatory blood pressure monitoring in male children receiving stimulant therapy. Ann Pharmacother 2002;36:1142-9.

9 Findling RL, Short EJ, Manos MJ. Short-term cardiovascular effects of methylphenidate and adderall. J Am Acad Child Adolesc Psychiatry 2001;40:525-9.

10 Winterstein AG, Gerhard T, Shuster J, Johnson M, Zito JM, Saidi A. Cardiac safety of central nervous system stimulants in children and adolescents with central nervous system stimulants in children and adolescents with

11 Graham J, Banaschewski T, Buitelaar J, Coghill D, Danckaerts M, Dittmann RW, et al. European guidelines on managing adverse effects of medication for ADHD. Eur Child Adolesc Psychiatry 2011;20:17-37.

12 Olfson M, Huang C, Gerhard T, Winterstein A, Crystal S, Allison P, et al. Stimulants and cardiovascular events in youth with attention-deficit/hyperactivity disorder. J Am Acad Adolesc Psychiatry 2012;51:147-56.

13 Schelleman H, Bilker WB, Strom BL, Kimmel SE, Newcomb C, Guevara JP, et al. Cardiovascular events and death in children exposed and unexposed to ADHD agents. Pediatrics 2011;127:1102-10.

14 Cooper WO, Habel LA, Sox CM, Chan KA, Arbogast PG, Cheetham TC, et al. ADHD drugs and serious cardiovascular events in children and young adults. $N$ Engl $\mathrm{J} \mathrm{Med}$ 2011;365:1896-904.

15 Chung CP, Murray KT, Stein CM, Hall K, Ray WA. A computer case definition for sudden cardiac death. Pharmacoepidemiol Drug Saf 2010;19:563-72.

16 Rosenbaum PR, Rubin DB. The central role of the propensity score in observational studies for causal effects. Biometrika 1983;70:41-55.

17 Harrell FE. Regression modeling strategies: with applications to linear models, logistic regression, and survival analysis. Springer, 2001.

18 Cox DR. Regression models and life-tables. J R Stat Soc Series B Stat Methodol 1972;34:187.

19 Kalbfleisch JD, Prentice RL. The statistical analysis of failure time data. Wiley, 1980.

20 Herndon JE, Harrell FE. The restricted cubic spline as baseline hazard in the proportional hazards model with step function time-dependent covariables. Stat Med 1995:14:2119-29.

21 Maldonado G, Greenland S. Simulation study of confounder-selection strategies. Am J Epidemiol 1993;138:923-36.

22 Cornfield J. A method of estimating comparative rates from clinical data; applications to cancer of the lung, breast, and cervix. J Natl Cancer Inst 1951;11:1269-75.

23 Zito JM, Safer DJ, dosReis S, Magder LS, Gardner JF, Zarin DA. Psychotherapeutic medication patterns for youths with attention-deficit/hyperactivity disorder. Arch Pediatr Adolesc Med 1999:153:1257-63.

24 Winterstein AG, Gerhard T, Shuster J, Zito J, Johnson M, Liu H, et al. Utilization of pharmacologic treatment in youths with attention deficit/hyperactivity disorder in Medicaid database. Ann Pharmacother 2008;42:24-31.

25 McCarthy SC, Potts N, Taylor L, Wong E. Mortality associated with attention-deficit hyperactivity disorder (ADHD) drug treatment: a retrospective cohort study of children, adolescents and young adults using the General Practice Research Database. Drug Safety 2009;32:1089-96.

26 Ray WA. Evaluating medication effects outside of clinical trials: new-user designs. Am J Epidemiol 2003;158:915-20

27 Chen CY, Gerhard T, Winterstein AG. Determinants of initial pharmacological treatment for youths with attention-deficithyperactivity disorder. J Child Adolesc Psychopharmacol 2009;19:187-95.

28 Ray WA, Meredith S, Thapa PB, Meador KG, Hall K, Murray KT. Antipsychotics and the risk of sudden cardiac death. Arch Gen Psychiatry 2001;58:1161-7.

29 Ray WA, Chung CP, Murray KT, Hall K, Stein CM. Atypical antipsychotic drugs and the risk of sudden cardiac death. N Engl J Med 2009;360:225-35.

30 Umana E, Solares CA, Alpert MA. Tachycardia-induced cardiomyopathy. Am J Med 2003;114:51-5.

31 El Hajiar M, Hermida JS, Caron FM, Nassif D, Bourges-Petit E, Maingourd Y. [Tachycardia-induced cardiomyopathy in children: a difficult diagnosis but a reversible form of left ventricular dysfunction.] Arch Pediatr 2004;11:24-8.

32 Shinbane JS, Wood MA, Jensen DN, Ellenbogen KA, Fitzpatrick AP, Scheinman MM. Tachycardia-induced cardiomyopathy: a review of animal models and clinical studies. $J$ Am Coll Cardiol 1997;29:709-15.

Accepted: 13 June 2012

\section{Cite this as: BMJ 2012;345:e4627}

This is an open-access article distributed under the terms of the Creative Commons Attribution Non-commercial License, which permits use, distribution, and reproduction in any medium, provided the original work is properly cited, the use is non commercial and is otherwise in compliance with the license. See: http://creativecommons.org/licenses/by$\mathrm{nc} / 2.0 /$ and http://creativecommons.org/licenses/by-nc/2.0/legalcode. 


\section{What is already known on this topic}

Case reports, concerns about a drug class effect, and a potential pharmacological pathway substantiated concerns about cardiac safety of central nervous system stimulants used in the treatment of children with psychiatric disorders

Only one of several observational studies was sufficiently powered to investigate the risk for major cardiovascular events and found no association

\section{What this study adds}

In a vulnerable population of children eligible for public insurance in the United States there was no significant association between use of stimulants for the treatment of mental health conditions and the outcomes of stroke, acute myocardial infarction, or sudden cardiac death

Even in children with congenital heart and other cardiac disease receiving stimulant treatment for conditions such as attention-deficit/hyperactivity disorder there was no increased cardiovascular risk, but study power was limited

Several additional sensitivity analyses confirmed no appreciable association between stimulant treatment and major cardiovascular events, but effect of long term use or long term consequences of even small increases in blood pressure or heart rate were not evaluated

\section{Tables}

Table 1/ Baseline sociodemographic characteristics in children and young people with mental health diagnosis according to use of stimulants. Figures are numbers (percentages), unless stated otherwise, with and without weighting for propensity score

Unweighted*

Did not use stimulants ( $n=833263)$ Used stimulants $(n=386584)$

Mean (SD) age (years)

Index year:

\begin{tabular}{|c|c|c|c|c|}
\hline 1999 & $55053(6.6)$ & $27262(7.1)$ & 6.8 & 6.6 \\
\hline 2000 & $99192(11.9)$ & 44489 (11.5) & 11.8 & 11.4 \\
\hline 2001 & $100506(12.1)$ & 48304 (12.5) & 12.2 & 11.8 \\
\hline 2002 & $101593(12.2)$ & 53565 (13.9) & 12.7 & 12.3 \\
\hline 2003 & $111810(13.4)$ & $59435(15.4)$ & 14.0 & 13.8 \\
\hline 2004 & $123943(14.9)$ & $64590(16.7)$ & 15.5 & 15.7 \\
\hline 2005 & $121772(14.6)$ & 52355 (13.5) & 14.3 & 14.8 \\
\hline 2006 & $119394(14.3)$ & $36584(9.5)$ & 12.8 & 13.7 \\
\hline \multicolumn{5}{|l|}{ Race: } \\
\hline White & $459302(55.1)$ & $226469(58.6)$ & 56.20 & 56.95 \\
\hline Black & $239115(28.7)$ & $112102(29.0)$ & 28.77 & 27.66 \\
\hline American Indian & $5934(0.7)$ & $2197(0.6)$ & 0.66 & 0.66 \\
\hline Asian & $3200(0.4)$ & $808(0.2)$ & 0.33 & 0.35 \\
\hline Hispanic & $97723(11.7)$ & $33732(8.7)$ & 10.80 & 11.09 \\
\hline Hawaiian & $271(0.03)$ & $93(0.02)$ & 0.03 & 0.04 \\
\hline Mixed Hispanic & $6354(0.8)$ & $1383(0.4)$ & 0.63 & 0.61 \\
\hline Multiracial & $756(0.09)$ & $282(0.1)$ & 0.08 & 0.08 \\
\hline Unknown & $20608(2.47)$ & $9518(2.5)$ & 2.49 & 2.55 \\
\hline Male & 443596 (53.2) & $270702(70.0)$ & 58.7 & 58.8 \\
\hline Foster care & $78806(9.5)$ & $29867(7.7)$ & 8.9 & 8.7 \\
\hline Disability & $12408(1.5)$ & $5149(1.3)$ & 1.5 & 1.5 \\
\hline Poverty & 90560 (10.9) & $38483(10.0)$ & 10.6 & 10.3 \\
\hline \multicolumn{5}{|l|}{ State: } \\
\hline Alabama & $1865(0.22)$ & $979(0.25)$ & 0.23 & 0.22 \\
\hline Arkansas & $24765(3.0)$ & 12275 (3.2) & 3.05 & 3.07 \\
\hline Florida & $54690(6.6)$ & $24493(6.3)$ & 6.49 & 6.42 \\
\hline Georgia & $55880(6.7)$ & $33012(8.5)$ & 7.30 & 7.31 \\
\hline lowa & $12202(1.5)$ & $5249(1.4)$ & 1.42 & 1.40 \\
\hline Idaho & $16495(2.0)$ & $4253(1.1)$ & 1.70 & 1.85 \\
\hline Illinois & 65994 (7.9) & 30917 (8.0) & 7.92 & 7.78 \\
\hline
\end{tabular}


Table 1 (continued)

\begin{tabular}{|c|c|c|c|c|}
\hline & \multicolumn{2}{|c|}{ Unweighted ${ }^{*}$} & \multicolumn{2}{|c|}{ Inverse weighted } \\
\hline & Did not use stimulants $(n=833263)$ & Used stimulants ( $n=386584)$ & Did not use stimulants & Used stimulants \\
\hline Indiana & 32729 (3.9) & $14367(3.7)$ & 3.87 & 3.96 \\
\hline Kansas & $12495(1.5)$ & $3996(1.0)$ & 1.35 & 1.37 \\
\hline Louisiana & $31671(3.8)$ & $37853(9.8)$ & 5.74 & 5.81 \\
\hline Massachusetts & $12938(1.6)$ & $5880(1.5)$ & 1.56 & 1.66 \\
\hline Minnesota & $7455(0.9)$ & $2151(0.6)$ & 0.79 & 0.82 \\
\hline Missouri & $33594(4.0)$ & $10825(2.8)$ & 3.63 & 3.52 \\
\hline Mississippi & $28257(3.4)$ & $12906(3.3)$ & 3.36 & 3.27 \\
\hline North Carolina & $64902(7.8)$ & $35856(9.3)$ & 8.19 & 7.87 \\
\hline Nebraska & $19338(2.3)$ & $4759(1.2)$ & 1.98 & 1.96 \\
\hline New Hampshire & $10168(1.22)$ & $2699(0.7)$ & 1.06 & 1.18 \\
\hline New Jersey & $4798(0.6)$ & $1529(0.4)$ & 0.52 & 0.47 \\
\hline New York & $54688(6.6)$ & $13672(3.5)$ & 5.59 & 5.21 \\
\hline Ohio & 72203 (8.7) & $29542(7.6)$ & 8.33 & 8.29 \\
\hline Pennsylvania & $18138(2.2)$ & $6646(1.7)$ & 2.03 & 2.03 \\
\hline South Carolina & $33944(4.1)$ & 22900 (5.9) & 4.62 & 4.50 \\
\hline Tennessee & $30462(3.7)$ & $12839(3.3)$ & 3.55 & 3.56 \\
\hline Texas & $92001(11.0)$ & $42689(11.0)$ & 11.11 & 11.66 \\
\hline Virginia & $6772(0.8)$ & $2492(0.6)$ & 0.76 & 0.79 \\
\hline Vermont & $10811(1.3)$ & $1948(0.5)$ & 1.05 & 1.15 \\
\hline Wisconsin & $11847(1.4)$ & $5112(1.3)$ & 1.40 & 1.50 \\
\hline West Virginia & $12161(1.5)$ & $4745(1.2)$ & 1.39 & 1.37 \\
\hline
\end{tabular}


Table 2| Baseline clinical characteristics in children and young people with mental health diagnosis according to use of stimulants. Figures are numbers (percentages) with and without weighting for propensity score

\begin{tabular}{|c|c|c|c|c|}
\hline & \multicolumn{2}{|c|}{ Unweighted ${ }^{*}$} & \multicolumn{2}{|c|}{ Inverse weighted } \\
\hline & $\begin{array}{c}\text { Did not use stimulants }(n=833 \\
\text { 263) }\end{array}$ & Used stimulants ( $n=386584$ ) & $\begin{array}{l}\text { Did not use } \\
\text { stimulants }\end{array}$ & Used stimulants \\
\hline \multicolumn{5}{|l|}{ High risk conditions: } \\
\hline Malign neoplasm & $2634(0.32)$ & $831(0.21)$ & 0.28 & 0.29 \\
\hline $\begin{array}{l}\text { Hereditary or acquired haemolytic and } \\
\text { aplastic anaemia }\end{array}$ & $2477(0.30)$ & $720(0.19)$ & 0.26 & 0.27 \\
\hline HIV/AIDS & $961(0.12)$ & $181(0.05)$ & 0.09 & 0.10 \\
\hline Congenital heart disease & $7085(0.85)$ & $2839(0.73)$ & 0.82 & 0.82 \\
\hline Cardiomyopathy & $279(0.03)$ & $56(0.01)$ & 0.03 & 0.02 \\
\hline Valve disorder & $1026(0.12)$ & $328(0.08)$ & 0.11 & 0.12 \\
\hline Cerebrovascular disease & $1198(0.14)$ & $428(0.11)$ & 0.13 & 0.14 \\
\hline \multicolumn{5}{|l|}{ Other cardiac risk factors: } \\
\hline Disease of central nervous system & $8616(1.03)$ & $2229(0.58)$ & 0.89 & 0.87 \\
\hline Liver/renal disease & $292(0.31)$ & $702(0.18)$ & 0.27 & 0.26 \\
\hline Smoking & $0(0.00)$ & $0(0.00)$ & 0.00 & 0.00 \\
\hline Atherosclerosis & $231(0.03)$ & $125(0.03)$ & 0.03 & 0.03 \\
\hline Hyperlipidaemia & $2395(0.29)$ & $803(0.21)$ & 0.27 & 0.30 \\
\hline Collagen vascular disorder & $1404(0.17)$ & $364(0.09)$ & 0.15 & 0.16 \\
\hline Obesity & $8733(1.05)$ & $2885(0.75)$ & 0.96 & 1.04 \\
\hline Diabetes mellitus & $936(0.11)$ & $310(0.08)$ & 0.10 & 0.11 \\
\hline Disease of arteries & $706(0.08)$ & $263(0.07)$ & 0.08 & 0.08 \\
\hline Disease of veins & $1079(0.13)$ & $308(0.08)$ & 0.11 & 0.12 \\
\hline Amino acid transport disorder & $1444(0.17)$ & $424(0.11)$ & 0.15 & 0.16 \\
\hline $\begin{array}{l}\text { Acute/chronic rheumatic, ischaemic, } \\
\text { pulmonary, or unspecified heart disease }\end{array}$ & $1684(0.20)$ & $547(0.14)$ & 0.18 & 0.18 \\
\hline Hypertension & $2613(0.31)$ & $555(0.14)$ & 0.26 & 0.27 \\
\hline Conduction disorders & $3323(0.40)$ & $1123(0.29)$ & 0.37 & 0.35 \\
\hline Heart failure & $232(0.03)$ & $51(0.01)$ & 0.02 & 0.02 \\
\hline Cardiac symptoms & $19330(2.32)$ & $4946(1.28)$ & 1.99 & 2.07 \\
\hline Immunodeficiency & $728(0.09)$ & $256(0.07)$ & 0.08 & 0.09 \\
\hline Convulsions & $14292(1.72)$ & $5657(1.46)$ & 1.66 & 1.65 \\
\hline Respiratory infections & $50227(6.03)$ & $25022(6.47)$ & 6.18 & 6.18 \\
\hline Asthma & $59355(7.12)$ & $31473(8.14)$ & 7.49 & 7.54 \\
\hline Lung disease & $3323(0.40)$ & $1072(0.28)$ & 0.36 & 0.33 \\
\hline \multicolumn{5}{|l|}{ Mental health diagnoses: } \\
\hline Anxiety & $65089(7.81)$ & $11915(3.08)$ & 6.33 & 6.94 \\
\hline Bipolar disorder & $7007(0.84)$ & $2224(0.58)$ & 0.77 & 0.83 \\
\hline Schizophrenia & $1792(0.22)$ & $271(0.07)$ & 0.17 & 0.20 \\
\hline Depression & $58487(7.02)$ & $12080(3.12)$ & 5.81 & 6.27 \\
\hline Autism & $5813(0.70)$ & $2330(0.60)$ & 0.68 & 0.74 \\
\hline Learning disabilities & $3922(0.47)$ & $1025(0.27)$ & 0.41 & 0.45 \\
\hline Tic disorder & $1558(0.19)$ & $667(0.17)$ & 0.19 & 0.22 \\
\hline \multicolumn{5}{|l|}{ Drugs: } \\
\hline Antidepressants & $48341(5.80)$ & $15003(3.88)$ & 5.28 & 5.77 \\
\hline Antipsychotics & $21560(2.59)$ & $7754(2.01)$ & 2.45 & 2.54 \\
\hline Anticonvulsants & $27196(3.26)$ & $8674(2.24)$ & 2.97 & 3.18 \\
\hline Anxiolytics & 24885 (2.99) & 12099 (3.13) & 3.05 & 3.16 \\
\hline Lithium & $1547(0.19)$ & $329(0.09)$ & 0.16 & 0.18 \\
\hline
\end{tabular}


Table 2 (continued)

\begin{tabular}{|c|c|c|c|c|}
\hline & \multicolumn{2}{|c|}{ Unweighted* } & \multicolumn{2}{|c|}{ Inverse weighted } \\
\hline & $\begin{array}{l}\text { Did not use stimulants }(n=833 \\
\text { 263) }\end{array}$ & Used stimulants ( $n=386584$ ) & $\begin{array}{l}\text { Did not use } \\
\text { stimulants }\end{array}$ & Used stimulants \\
\hline a agonists & $8521(1.02)$ & $9621(2.49)$ & 1.54 & 1.49 \\
\hline$\beta$ blocker & $2721(0.33)$ & $628(0.16)$ & 0.28 & 0.29 \\
\hline Calcium channel blocker & $486(0.06)$ & $82(0.02)$ & 0.05 & 0.05 \\
\hline $\begin{array}{l}\text { Angiotensin converting enzyme (ACE) } \\
\text { inhibitors }\end{array}$ & $1652(0.20)$ & $290(0.08)$ & 0.16 & 0.16 \\
\hline Diuretics & $1114(0.13)$ & $224(0.06)$ & 0.11 & 0.11 \\
\hline Statins/fibrates & $269(0.03)$ & $69(0.02)$ & 0.03 & 0.03 \\
\hline Nitrate & $38(0.00)$ & $17(0.00)$ & 0.00 & 0.00 \\
\hline Antiarrhythmics & $518(0.06)$ & $122(0.03)$ & 0.05 & 0.05 \\
\hline Digoxin & $460(0.06)$ & $113(0.03)$ & 0.05 & 0.04 \\
\hline
\end{tabular}

${ }^{*} \mathrm{P}<0.001$ for all comparisons of exposed versus unexposed (unweighted), except atherosclerosis, disease of arteries, tic disorder, and nitrate use. 
Table 3| Adjusted odds ratios for primary and secondary study endpoints associated with stimulant use in children and young people with mental health diagnosis: full study cohort and low risk and high risk groups, full cohort with injury censoring, and stimulant users (current versus former use)

No of events

Primary endpoint: sudden cardiac death, AMI, stroke

\begin{tabular}{lll}
\hline Full cohort (current versus non-use) & 66 & 0.62 (0.27 to 1.44$)$ \\
\hline Low risk & 40 & 0.49 (0.16 to 1.48$)$ \\
\hline High risk & 26 & $1.02(0.28$ to 3.69$)$ \\
\hline With injury censoring (full cohort) & 38 & 0.66 (0.22 to 1.99$)$ \\
\hline Current $v$ former use (stimulant users only) & 13 & 1.07 (0.36 to 3.21$)$
\end{tabular}

Secondary endpoint: sudden cardiac death, AMI, stroke, ventricular arrhythmia

\begin{tabular}{lll}
\hline Full cohort (current versus non-use) & 95 & 0.74 (0.38 to 1.46$)$ \\
\hline Low risk & 60 & $0.52(0.21$ to 1.29$)$ \\
\hline High risk & 35 & 1.56 (0.56 to 4.34) \\
\hline With injury censoring (full cohort) & 54 & 0.62 (0.24 to 1.65$)$ \\
\hline Current $v$ former use (stimulant users only) & 20 & 1.17 (0.48 to 2.83) \\
\hline
\end{tabular}

AMl=acute myocardial infarction.

*Adjusted for propensity score and use of antipsychotics. 
Table 4| Unadjusted and adjusted event rates per 100000 patient years associated with stimulant use in children and young people with mental health diagnosis

\begin{tabular}{|c|c|c|c|c|}
\hline & \multicolumn{2}{|c|}{ Primary endpoint ${ }^{\star}$} & \multicolumn{2}{|c|}{ Secondary endpoint $†$} \\
\hline & Unadjusted & Adjusted¥ & Unadjusted & Adjusted¥ \\
\hline \multicolumn{5}{|l|}{ Full cohort: } \\
\hline Non-use & 3.5 (2.7 to 4.6$)$ & Reference & 5.0 (4.0 to 6.2 ) & Reference \\
\hline Current use & 1.7 (0.8 to 3.5$)$ & $2.2(1.0$ to 5.1$)$ & 2.6 (1.5 to 4.8$)$ & 3.7 (1.9 to 7.3$)$ \\
\hline Former use & $1.5(0.7$ to 3.4$)$ & $2.0(0.8$ to 4.9$)$ & 2.3 (1.2 to 4.3$)$ & 3.2 (1.5 to 6.5$)$ \\
\hline \multicolumn{5}{|c|}{ Low risk cohort: } \\
\hline Non-use & 2.1 (1.5 to 3.0$)$ & Reference & 3.1 (2.3 to 4.2 ) & Reference \\
\hline Current use & $1.0(0.4$ to 2.6$)$ & $1.0(0.3$ to 3.1$)$ & 1.5 (0.7 to 3.3$)$ & $1.6(0.7$ to 4.0$)$ \\
\hline Former use & $1.3(0.5$ to 3.1$)$ & 1.5 (0.6 to 4.0$)$ & $2.0(1.0$ to 4.1$)$ & 2.4 (1.1 to 5.2$)$ \\
\hline \multicolumn{5}{|c|}{ High risk cohort: } \\
\hline Non-use & 75.2 (49.5 to 114.1$)$ & Reference & 99.1 (68.9 to 142.6 ) & Reference \\
\hline Current use & 51.5 (16.6 to 159.7$)$ & 76.7 (21.0 to 277.3 ) & 85.8 (35.7 to 206.2) & 154.6 (55.5 to 430.0$)$ \\
\hline Former use & 17.0 (2.4 to 120.5$)$ & 24.1 (3.1 to 183.4 ) & 17.0 (2.4 to 120.5$)$ & 27.7 (4.0 to 208.1 ) \\
\hline \multicolumn{5}{|c|}{ Injury censored cohort: } \\
\hline Non-use & 2.4 (1.7 to 3.4$)$ & Reference & 3.4 (2.6 to 4.6$)$ & Reference \\
\hline Current use & $1.2(0.5$ to 3.3$)$ & $1.6(0.5$ to 4.8$)$ & 1.5 (0.6 to 3.7$)$ & 2.1 (0.8 to 5.7$)$ \\
\hline Former use & $1.0(0.3$ to 3.0$)$ & $1.3(0.4$ to 4.3$)$ & $1.6(0.7$ to 3.9$)$ & $2.2(1.1$ to 4.5$)$ \\
\hline
\end{tabular}

*Sudden cardiac death, acute myocardial infarction, stroke.

†Sudden cardiac death, acute myocardial infarction, stroke, ventricular arrhythmia

$\ddagger$ Calculated by multiplying crude event rate of non-users by respective adjusted odds ratios. 


\section{Figure}

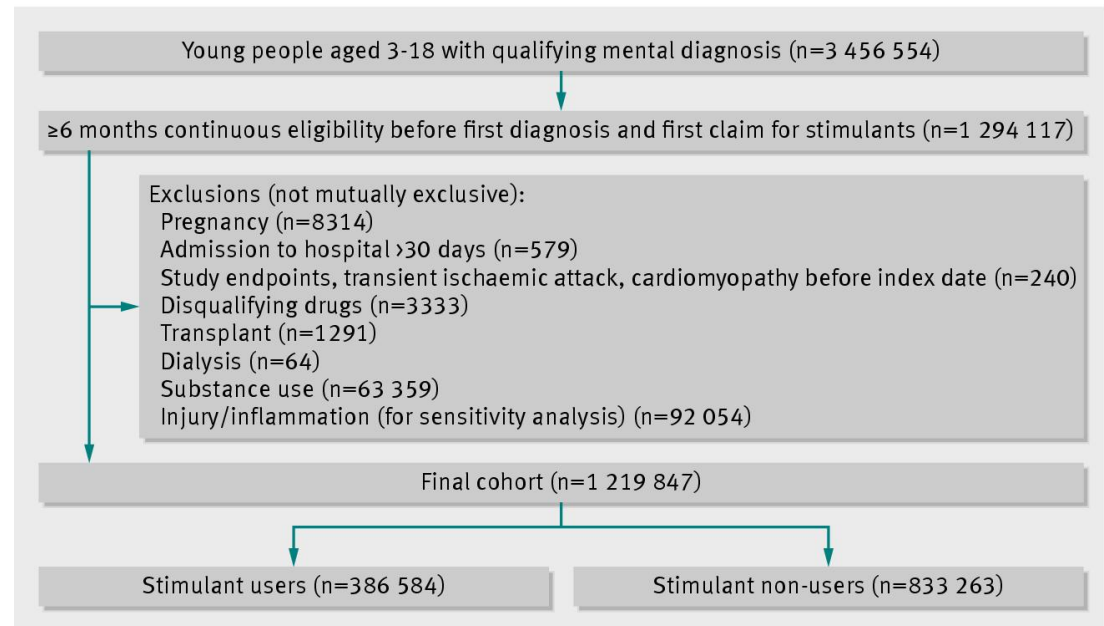

Study enrolment and details of exclusions 